\title{
Dependence of nano-confined surface condensation on tangentially external force field
}

\author{
Jin Huan PUa, Qiang SHENG ${ }^{a}$, Jie SUN ${ }^{b *}$, Wen WANGa and Hua Sheng WANGa* \\ ${ }^{a}$ School of Engineering and Materials Science, Queen Mary University of London, London E1 4NS, UK \\ ${ }^{\mathrm{b}}$ School of Chemical Engineering and Technology, Xi'an Jiaotong University, Xi'an, Shaanxi 710049, China
}

\begin{abstract}
Molecular dynamics (MD) simulations were conducted to investigate the dependence of nano-confined surface condensation on tangentially external force filed. The dynamic behaviors of surface condensation were simulated on a smooth solid surface with fixed wettability (denoted by $\beta$ ) and different external force fields (denoted by $f_{\mathrm{e}}$ ), and on surfaces with different $\beta$ and fixed $f_{\mathrm{e}}$. The heat transfer analysis shows that $f_{\mathrm{e}}$, as a bulk factor, indirectly influences the interfacial thermal resistance $(R)$ by direct influence on surface condensation resulting from the viscous and frictional dissipated heats. This is because the dissipated heats result in superheat of the vapor, leading to the delay or elimination of onset of surface condensation. This finding extends the general understanding that $R$ is only dependent on the interfacial factors, such as $\beta$ and surface topology. The energy balance analysis shows that, for condensation cases, the largest proportion of the heat transferred through the fluidsolid interface is attributed to the change in the internal energy, while for non-condensation cases, it is attributed to the dissipated heats due to $f_{\mathrm{e}}$. As $f_{\mathrm{e}}$ increases or $\beta$ decreases, the dissipated heats increase and gradually take over the total heat transferred from fluid to solid, which finally reduces or suppresses the occurrence of surface condensation.
\end{abstract}

Keywords: Nano-confined surface condensation; Molecular dynamics simulation;

External force field; Energy conversion analysis; Heat transfer

\section{Introduction}

The understanding of the physical behavior of nano-confined fluids has drawn much attention due to its potential applications such as in chemistry, nanomaterials, drug delivery,

*Authors to whom correspondence should be addressed. Electronic mails: sunjie@xjtu.edu.cn (JS); h.s.wang@qumul.ac.uk (HSW). 
nanoelectronic devices [1]-[5]. Phase change and fluid flow play important roles in these applications [6]. In nanoscale, surface effects dominate the flow and phase characteristics due to the large ratio of surface area to volume, leading to different mechanisms in comparison of those in macroscale [7]. Vapor condensation is a common physical phenomenon and appears two different modes, namely dropwise condensation (DWC) and filmwise condensation (FWC). The formation of initial droplet in DWC consists of three over-lapping stages i.e. the formation of clusters, generation of nuclei and emergence of nanoscale droplets. Molecular dynamics (MD) simulation has been widely used to investigate the insights into the phenomena of fluid flow and heat transfer in nanoscale. MD simulations [9]-[16] have demonstrated features during the onset, early and developed stages of surface condensation. In the case of condensation in a slit pore, the rate of nucleation is higher than that in the homogenous vapor at the same vapor density and temperature, irrespective of the strength of attraction between the wall and vapor molecules [9]. The nucleation is more likely to initiate on the high energy particles that embedded in a low energy surface and clusters do not migrate around their original positions [11]. It has also been found that there are two different types of formation of nanoscale droplet depending on the relative surface free energy, i.e. the formations with and without film-like condensate, which enriches the 'classical hypotheses' of the onset of dropwise condensation [14]. For the formation with film-like condensate case, a film-like condensate is formed firstly when the surface with relatively large wettability is cooled, then it contracts and ruptures into several nuclei and finally evolves into a large droplet. During condensation there exists a competition between the interfacial thermal resistance and condensate bulk thermal resistance [15]. At the onset of condensation, the interfacial thermal resistance takes over, while the condensate bulk thermal resistance gradually dominates with condensate thickness growing. 
MD simulations have also been used to investigate the behavior of nano-confined fluid flow [17]-[20]. Their results show that the velocity slip and temperature jump at the solid-fluid interface are significant and the behavior of confined fluid can be largely controlled by appropriately modifying the boundary and bulk factors. Their results also show that in nanoconfined fluid flow the dissipated heat, resulting from viscous friction of fluid and interfacial friction at fluid-solid interface, is an important factor. In practice surface condensation occurs under external force filed and its effect needs to be taken into account, where fluid flow and heat transfer are coupled. MD simulations [21] investigated features of surface condensation under external force field but mainly focused on how the composite nano-surface to sustain and enhance condensation heat transfer as well as the enhancement mechanism from the thermal resistance point of view.

So far no detailed investigations have been done on the surface condensation of nano-confined fluids under external force filed. In this work, we will investigate the effect of external force field on nano-confined surface condensation using MD simulation. Owing to the strong effects of nanoscale surfaces, the surface wettability is also taken into account. Moreover, attentions in MD simulations of condensation have been mainly paid to cluster analysis [22], formation free energy [23], computational strategies [24],[25], surface properties [11] and so on. In this work, we will quantitatively analyze the energy balance and look at the mechanism of the effects of external force filed from energy conversion point of view. Our work helps further understand the fundamental of practical nano-confined surface condensation and potentially offers a new way for artificial control and enhancement of surface condensation.

\section{Computational methods}

The surface condensation with external force field is of concern in the present work using MD simulation (see Fig. 1). All the simulations are conducted using LAMMPS [26] (large-scale atomic/molecular massively parallel simulator) software package. The overall size of 
simulation box measures $l_{x} \times l_{y} \times l_{z}=588.45 \times 39.23 \times 470.76 \AA$. Owing to symmetry, this simulation domain is just half of the nanochannel. Therefore, in the $y$ and $z$ directions, periodic boundary conditions are used, while in the $x$ direction, fixed boundary condition is employed and reflection boundary condition is also applied at the rightmost end. The solid wall constructed by Pt-like atoms is arranged on the leftmost region of the simulation box and the rest region is occupied by the Lennard-Jones (L-J) fluid of Ar. The 12-6 L-J potential function is employed for fluid-fluid interaction:

$$
\varphi(r)=4 \varepsilon\left[\left(\frac{\sigma}{r}\right)^{12}-\left(\frac{\sigma}{r}\right)^{6}\right]
$$

where $r$ is the intermolecular separation, $\varepsilon$ and $\sigma$ are the energy and length characteristic parameters, respectively. The potential function is truncated at the cut-off radius $r_{\mathrm{c}}=3.5 \sigma$, beyond which molecular interactions are ignored. $\varepsilon_{\mathrm{Ar}-\mathrm{Ar}}=0.01040 \mathrm{eV}$ and $\sigma_{\mathrm{Ar}-\mathrm{Ar}}=3.405 \AA$ are used. The solid wall is represented by three layers of Pt-like atoms arranged as a face-centered cubic (FCC) lattice and the length of the unit cell is $3.926 \AA$. Two extra layers of Pt-like atoms are fixed on the leftmost region of the solid wall to serve as a frame. The interaction between solid atoms is L-J type only with $\varepsilon$ Pt-Pt $=0.521875 \mathrm{eV}$ and $\sigma_{\mathrm{Pt}-\mathrm{Pt}}=2.475 \AA$. The fluid-solid interaction is also governed by Eq. (1) but with different energy and length parameters, i.e. $\varepsilon_{\mathrm{Ar}-}$ $\mathrm{Pt}=\beta \varepsilon_{\mathrm{Ar}-\mathrm{Ar}}$ and $\sigma_{\mathrm{Ar}-\mathrm{Pt}}=0.91 \sigma_{\mathrm{Ar}-\mathrm{Ar}}$, where $\beta$ is fluid-solid bonding strength parameter indicating the surface free energy, or equivalently the surface wettability, because normally higher surface free energy suggests stronger surface wettability. All the simulations are performed in three stages with a time step of $5 \mathrm{fs}$. In stage 1 (equilibrium stage), the saturated vapor molecules at $T_{\mathrm{v}}=120 \mathrm{~K}$ are uniformly arranged. The initial $3 \mathrm{~ns}$ allows the system to reach the thermal equilibrium state at $120 \mathrm{~K}$, where the Langevin thermostat is used to maintain the temperature of both the wall and fluid. In stage 2 (steady-state stage), an external force is applied on each fluid molecule in the $z$-direction. Meanwhile, the thermostat applied on argon is removed and 
only the NVE ensemble is employed for argon. The temperature control of the solid wall is still maintained at $120 \mathrm{~K}$. The following $30 \mathrm{~ns}$ guarantees the system to reach a steady state, after which another $5 \mathrm{~ns}$ is employed to sample the properties, such as velocity and temperature. In stage 3 (condensation/cooling stage), the condensation process is triggered by suddenly reducing the surface temperature to $90 \mathrm{~K}$ and maintained afterwards for a period of $20 \mathrm{~ns}$ by the Langevin thermostat. To investigate the effects of external force field on condensation over surfaces with different free energies, two steps are carried out. In step one, the effect of external force field on condensation is primarily investigated with different external force $\left(f_{\mathrm{e}}\right)$ in the $z$ direction from $0.0001 \varepsilon \sigma^{-1}$ to $0.0007 \varepsilon \sigma^{-1}$ ( $\varepsilon$ and $\sigma$ employ Ar parameters.) and fixed $\beta$ equal to 0.35. In step two, the effect of surface free energy on condensation is investigated with fixed $f_{\mathrm{e}}$ equal to $0.0005 \varepsilon \sigma^{-1}$ and different $\beta$ from 0.15 to 0.75 . In order to conveniently evaluate the external force fields, Tab. 1 gives the exact values in real unit.

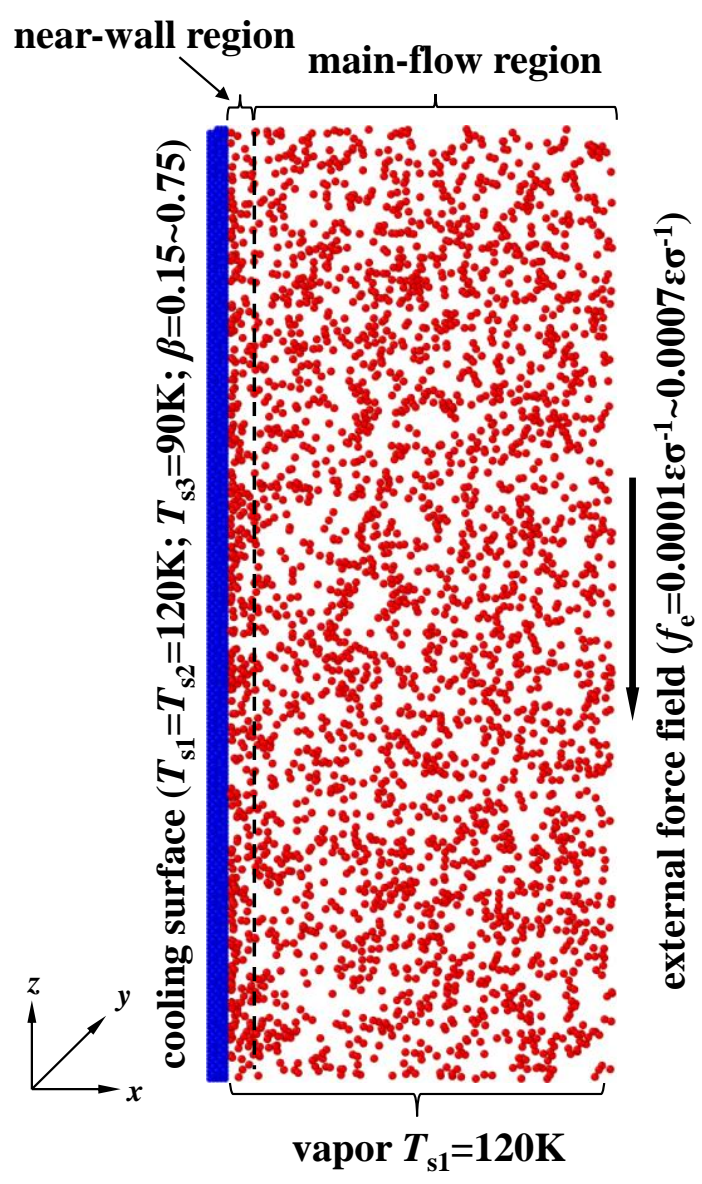


Figure 1. Schematic of computational model. The size of simulation box is $l_{x} \times l_{y} \times l_{z}=588.45 \times 39.23 \times 470.76$ A. Solid atoms are in blue and fluid molecules are in red.

Table 1. External forces exerted on each fluid molecular in the present simulations.

\begin{tabular}{lccccccc}
\hline \hline$f_{\mathrm{e}} / \mathrm{eV} \cdot \AA^{-1}$ & $0.0001 \varepsilon \sigma^{-1}$ & $0.0002 \varepsilon \sigma^{-1}$ & $0.0003 \varepsilon \sigma^{-1}$ & $0.0004 \varepsilon \sigma^{-1}$ & $0.0005 \varepsilon \sigma^{-1}$ & $0.0006 \varepsilon \sigma^{-1}$ & $0.0007 \varepsilon \sigma^{-1}$ \\
\hline$f_{\mathrm{e}} / 10^{-15} \mathrm{~N}$ & 0.4887 & 0.9774 & 1.4661 & 1.9548 & 2.4435 & 2.9322 & 3.4209 \\
\hline \hline
\end{tabular}

\section{Results and discussion}

\subsection{Condensation dynamics}

\subsubsection{On surface with fixed wettability under different external force fields}

In stage 2 , the vapor molecules reach a steady state under different $f_{\mathrm{e}}$. With fixed surface wettability $(\beta=0.35)$, the velocity in the $\mathrm{z}$ direction $\left(v_{z}\right)$ and temperature profiles, shown in Fig. 2, are found to be sensitive to $f_{\mathrm{e}}$. When $f_{\mathrm{e}}$ increases, velocity gradient along the $x$ direction rises, and the apparent slippage appears and increases at the solid-vapor interface. Correspondingly, temperature of the vapor increases, which allows the saturated vapor to become superheated and the degree of superheat increases. Meanwhile, the temperature jump and gradient along the $x$ direction become larger with increasing $f_{\mathrm{e}}$. From the top views, as shown in Fig. 3(a), it is indicated that there is no significant difference of first layer of the liquid molecules between $f_{\mathrm{e}}=0$ and $f_{\mathrm{e}}=0.0007 \varepsilon \sigma^{-1}$ cases. This is also confirmed by the detailed quantitative results shown in Fig. 3(b) that the differences of density profiles under different $f_{\mathrm{e}}$ are generally small. Specifically, it can be seen, from the locally amplified inset in Fig.3(b), that the averaged density adjacent to the solid wall decreases from $0.0020 \AA^{-3}$ to $0.0011 \AA^{-3}$ with increasing $f_{\mathrm{e}}$ from 0 to $0.0007 \varepsilon \sigma^{-1}$ due to the increase of fluid temperature resulted from the dissipated heat. 


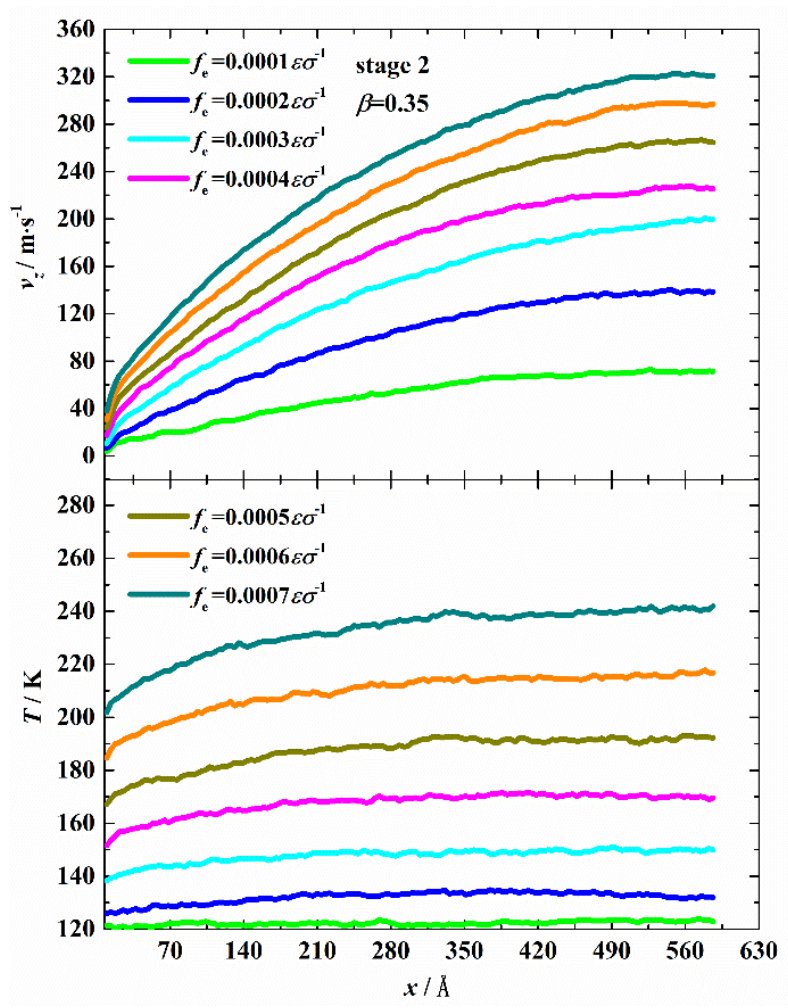

Figure 2. Velocity and temperature profiles of vapor molecules with $\beta=0.35$ under different external force on stage 2.

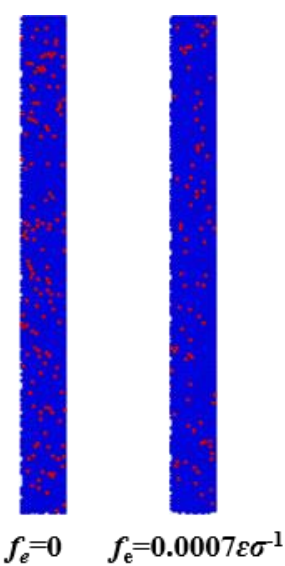

(b)

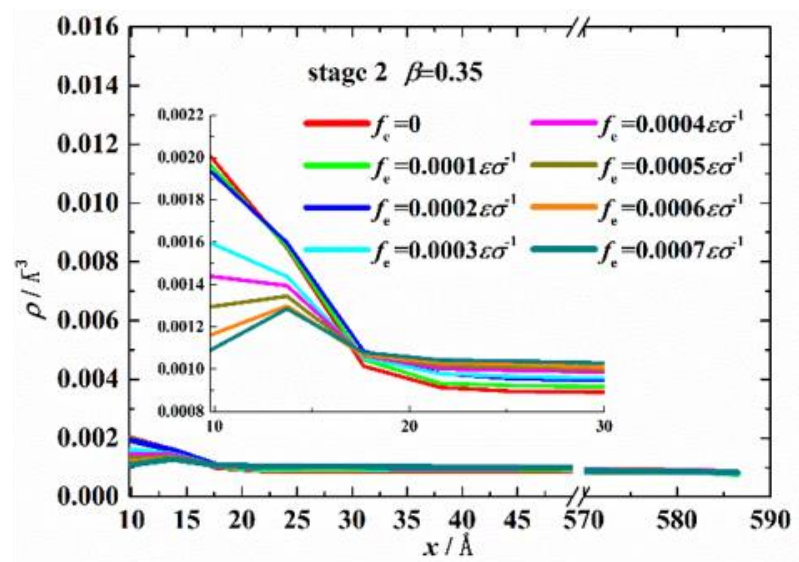

Figure 3. (a) Top view of first layer molecules adjacent to the solid wall. (b) Density profiles of vapor molecules with $\beta=0.35$ under different external force on Stage 2 .

In stage 3, when the surface temperature suddenly drops from $120 \mathrm{~K}$ to $90 \mathrm{~K}$, the vapor molecules are immediately cooled by the wall surface. The transient density profiles in the $x$ direction under different $f_{\mathrm{e}}$ are given in Fig.4. It can be clearly seen that in the cases with $f_{\mathrm{e}} \geq$ $0.0005 \varepsilon \sigma^{-1}$, the density profiles during stage 3 rarely vary, which suggests that there is no surface condensation. This can also be supported by the animation of simulation system (see supplementary material at [27] for Figs. S1-S3). For those condensation cases $\left(f_{\mathrm{e}}<0.0005 \varepsilon \sigma^{-}\right.$ 
${ }^{1}$ ), the condensate on the wall surface growing thicker is clearly indicated by the liquid density peaking and expanding as time evolves. Eventually, $f_{\mathrm{e}}=0,0.0001 \varepsilon \sigma^{-1}$ and $0.0002 \varepsilon \sigma^{-1}$ cases shape similarly in the density profile while $f_{\mathrm{e}}=0.0003 \varepsilon \sigma^{-1}$ and $0.0004 \varepsilon \sigma^{-1}$ cases shape another. By observing the animation of condensation under different $f_{\mathrm{e}}$ (see supplementary material at [27] for Figs. S4-S8), it can be found that similar density profiles are due to the same number of primary droplets. For $f_{\mathrm{e}}=0,0.0001 \varepsilon \sigma^{-1}$ and $0.0002 \varepsilon \sigma^{-1}$ cases, finally there are two primary droplets during stage 3, while for $f_{\mathrm{e}}=0.0003 \varepsilon \sigma^{-1}$ and $0.0004 \varepsilon \sigma^{-1}$ cases, there is only one. Fig. 5 shows the transient snapshots $(t=2,4,6,8,10,12$ and $14 \mathrm{~ns})$ for three representative cases $\left(f_{\mathrm{e}}\right.$ $=0,0.0002 \varepsilon \sigma^{-1}$ and $\left.0.0004 \varepsilon \sigma^{-1}\right)$. No matter for $f_{\mathrm{e}}=0$ or other cases, primary droplets form both through three over-lapping stages. Initially, obvious clusters are observed to deposit on the wall surface. Then, some clusters randomly migrate and coalesce with other clusters to form nuclei while the others disappear. Finally, a primary droplet emerges with upsizing by condensation of vapor molecules and numerous coalescences between those surviving nuclei. However, there are significant differences between these cases. Combining Figs. 4 and 5 together, it is clearly found that the existence of $f_{\mathrm{e}}$ can postpone the appearance of condensation when $f_{\mathrm{e}}$ is beyond to a certain threshold $\left(f_{c r}=0.0005 \varepsilon \sigma^{-1}\right.$ for surface with $\left.\beta=0.35\right)$. In the $f_{\mathrm{e}}=0$ case, obvious clusters can be observed at the initial time, while in the $f_{\mathrm{e}}=0.0004 \varepsilon \sigma^{-1}$ case, they only do after $4 \mathrm{~ns}$ (see Fig. 5). In addition, the densities for $f_{\mathrm{e}}=0.0003 \varepsilon \sigma^{-1}$ and $0.0004 \varepsilon \sigma^{-1}$ cases are apparently lower than those for $f_{\mathrm{e}}=0,0.0001 \varepsilon \sigma^{-1}$ and $0.0002 \varepsilon \sigma^{-1}$ cases, which suggests that the condensation intensity decreases with increasing $f_{\mathrm{e}}$. The essential reason is that $f_{\mathrm{e}}$ causes the dissipated heating effect, which further leads to the locally superheating state of vapor in vicinity of the wall surface. Due to the fact, after the surface temperature drops, the superheated vapor needs to be cooled to saturation state first before condensing, the strong dissipated heating effect greatly prevents the vapor molecules from condensing. The larger $f_{\mathrm{e}}$, the larger degree of superheating and harder the condensation could occur. It can also be seen that even 
small droplets start to move downward after formation with larger $f_{\mathrm{e}}$, which facilitates the coalescence between them and causes less primary droplets to emerge. For example, as shown in Fig. 5 , three primary droplets can be seen at $10 \mathrm{~ns}$ in $f_{\mathrm{e}}=0$ case while only two form in $f_{\mathrm{e}}=$ $0.0002 \varepsilon \sigma^{-1}$ case.

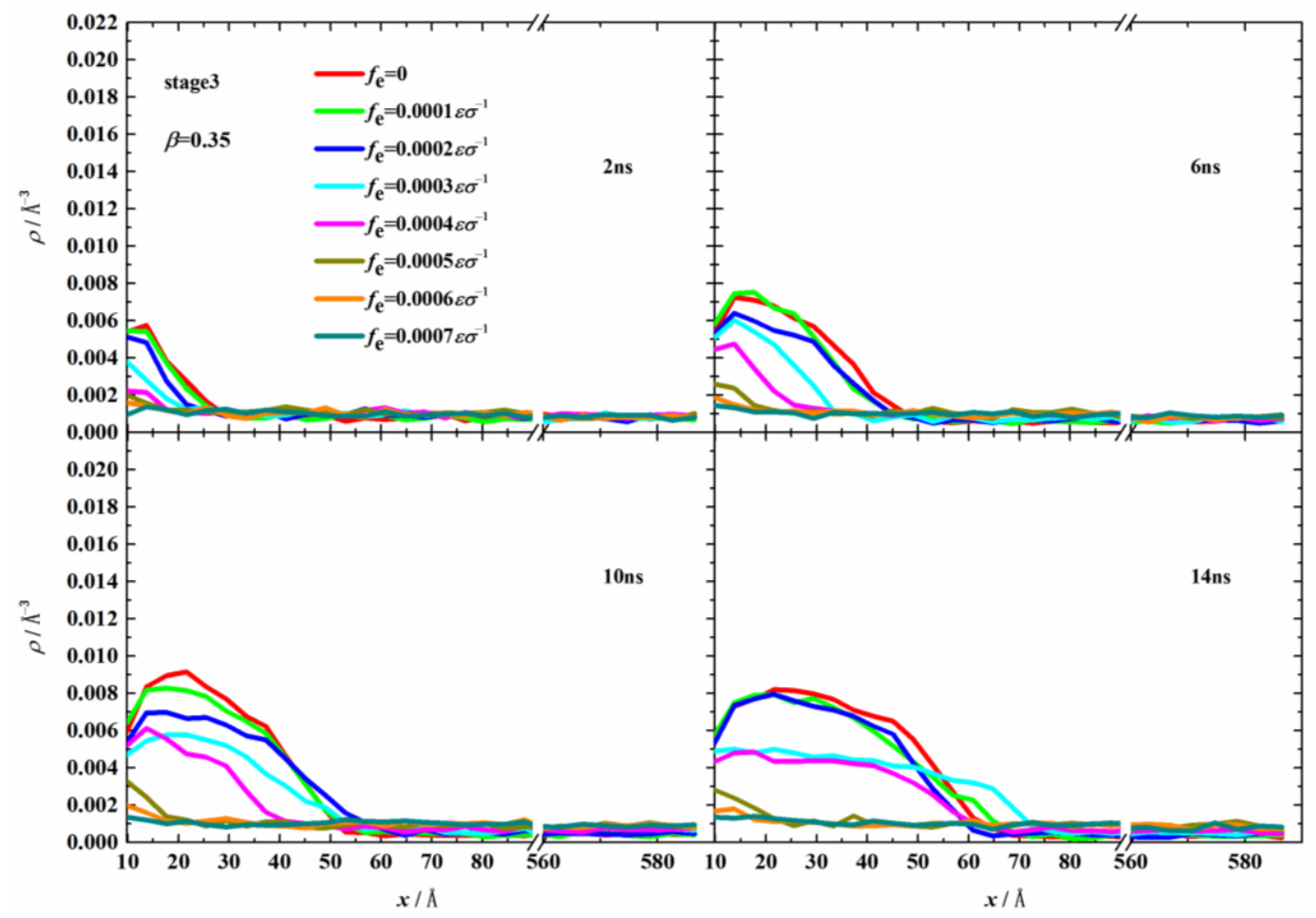

Figure 4. Transient density profiles with $\beta=0.35$ and different $f_{\mathrm{e}}$ during stage 3 . 


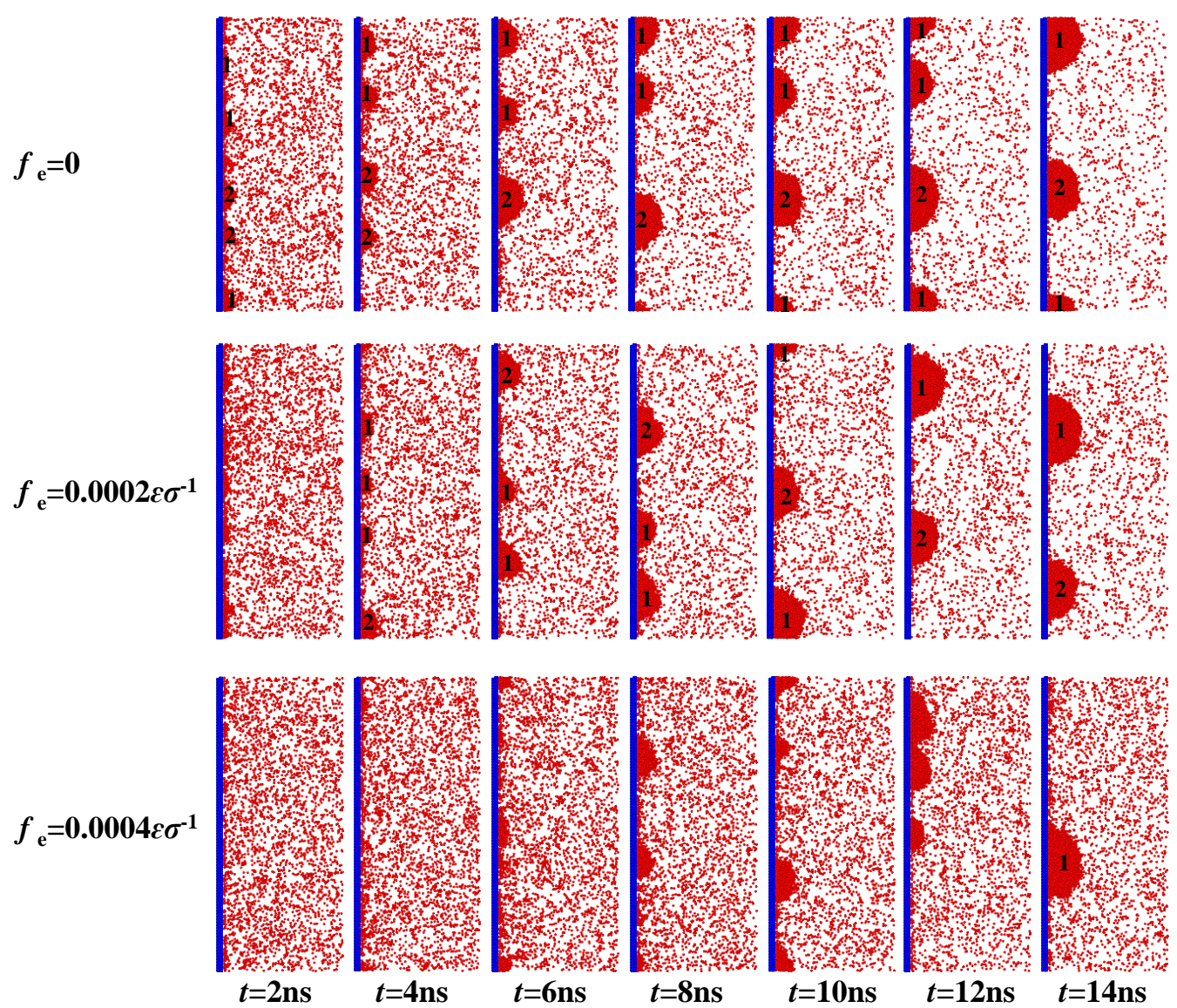

Figure 5. Transient snapshots with $\beta=0.35$ and different $f_{\mathrm{e}}$ during stage 3 .

\subsubsection{On surfaces with different wettabilities under fixed external force field}

In Fig. 6, based on whether there exists the velocity slippage under $f_{\mathrm{e}}=0.0005 \varepsilon \sigma^{-1}$, velocity profiles in the $\mathrm{z}$ direction $\left(v_{z}\right)$ are shown separately and different variations are observed. When $\beta$ is small $(\beta \leq 0.45)$, the velocity slip appears and decreases with increasing $\beta$. The variation near the main stream is not significant. All these lead to the velocity gradient along the $x$ direction increases with increasing $\beta$. Correspondingly, the temperature profile drops as a whole, whereas the temperature gradient tends to become steeper with increasing $\beta$. When $\beta$ is large $(\beta>0.45)$, the slippage disappears and the difference in velocity profiles is not conspicuous, which results from stronger solid-fluid interaction. Consequently, the increase in the fluid temperature is not dramatic and the difference between different $\beta$ is small when $\beta>$ 0.45 compared with small $\beta$ cases. As for density profiles shown in Fig.7, when $\beta$ increases, suggesting stronger solid-fluid interaction, the density adjacent to solid wall surface increases 
obviously. It can also be clearly seen from the top view of the first layer of fluid molecules adjacent to the solid wall surface, as shown in Fig. 7 (b). On surface with $\beta=0.15$, the adsorbed fluid molecules are few and isolated to each other, while as $\beta$ increases to 0.75 , the wall surface is almost fully occupied by massive fluid molecules.

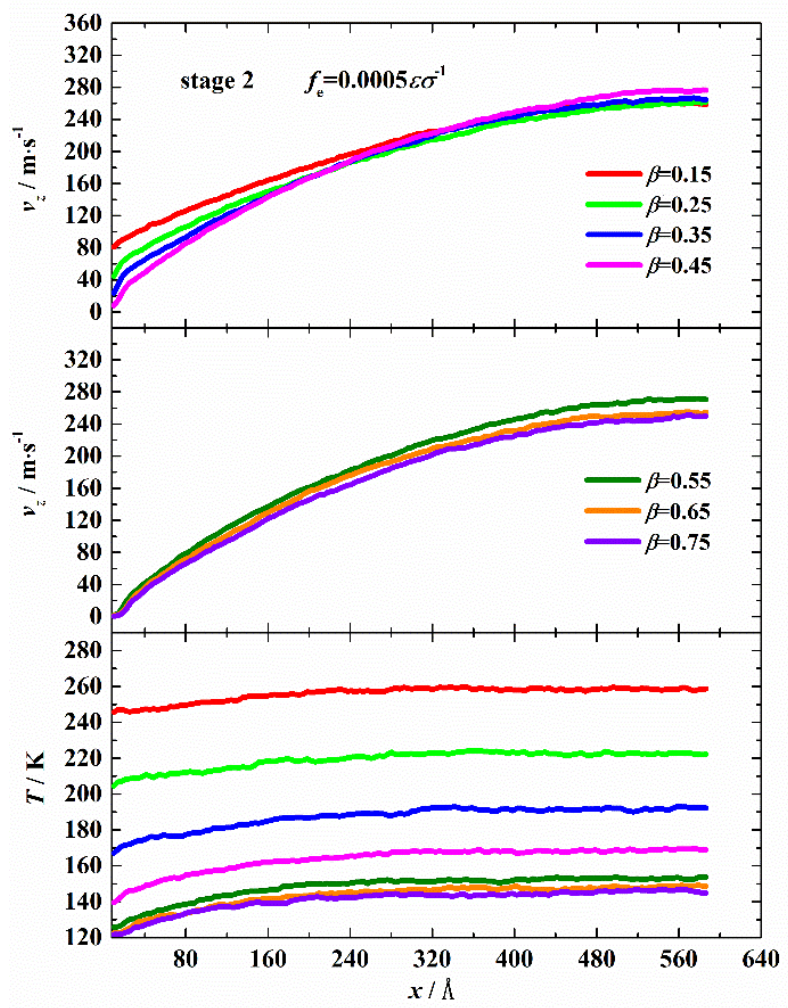

Figure 6. Velocity and temperature profiles of vapor molecules with different $\beta$ under $f_{\mathrm{e}}=0.0005 \varepsilon \sigma^{-1}$ on stage 2 .

(a)

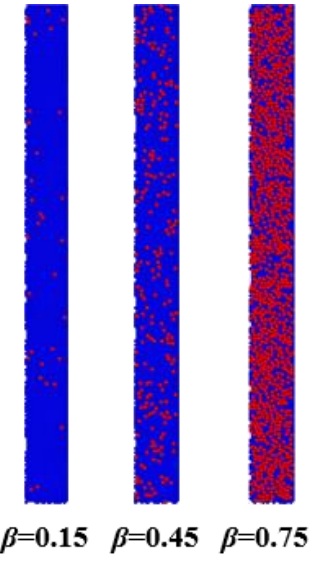

(b)

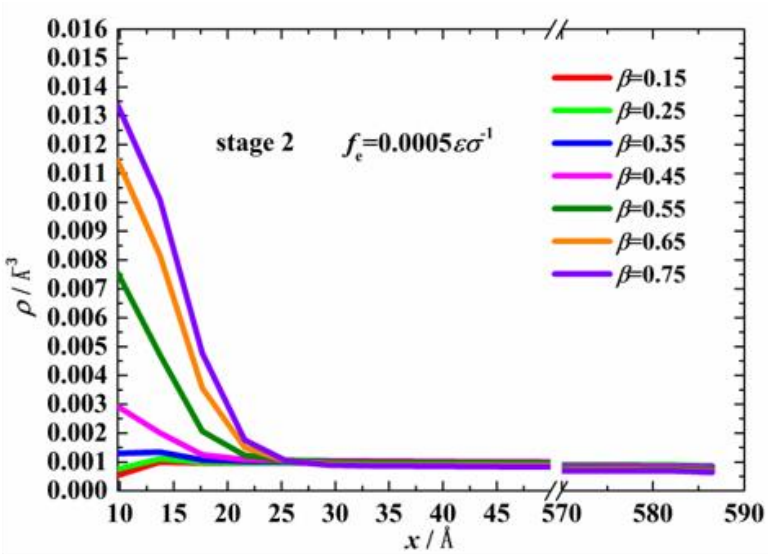

Figure 7. (a) First layer of molecules adjacent to solid wall (top view). (b) Density profiles of fluid molecules with different $\beta$ under $f_{\mathrm{e}}=0.0005 \varepsilon \sigma^{-1}$ in stage 2 .

From Fig. 8, it is seen that there are eventually three different kinds of density profiles in stage

3. When $\beta<0.45$, the density profiles do not increase and vary significantly, which indicates 
that the condensate bulk hardly forms due to the existence of external force field and weak solid-fluid interaction. When $\beta=0.45$, the density increases and the condensate grows thicker with time before $t=6 \mathrm{~ns}$. Comparatively, at $t=10 \mathrm{~ns}$, although the condensate becomes thicker significantly, the density drops. This can be explained by the transient snapshots of condensation shown in Fig. 8. It is seen that the film-like condensate grows continuously covering most of the wall surface at the initial time (see Fig. 8 at $t=2 \mathrm{~ns}$ ). Therefore, the density increases and the condensate grows thicker. Then, the film-like condensate starts to contract and rupture. After rupturing, obvious discrete droplets appear and start to coalesce. Due to the existence of external force, the droplets move downward and coalesces with a primary droplet. As the primary droplet forms and grows large enough, the droplet moving becomes much faster. The droplet forms an asymmetric cap-like shape, of which the front part is denser. Consequently, the density distribution tends to flatten. When $\beta>0.45$, the solid-fluid interaction is sufficiently strong so that numerous clusters instantaneously deposit on the wall surface when cooled and the surface is fully occupied by fluid molecules immediately. As can be seen in Figs. 8 and $9(t=2 \mathrm{~ns})$, the condensate film already becomes thick. With time evolving, the condensate film continues to grow thicker but the growth rate decreases and tends to cease at $t=10 \mathrm{~ns}$. As the condensate film grows thick enough, it starts to move downward slowly with noticeable fluctuation at the liquid-vapor interface due to the strong frictional force. 


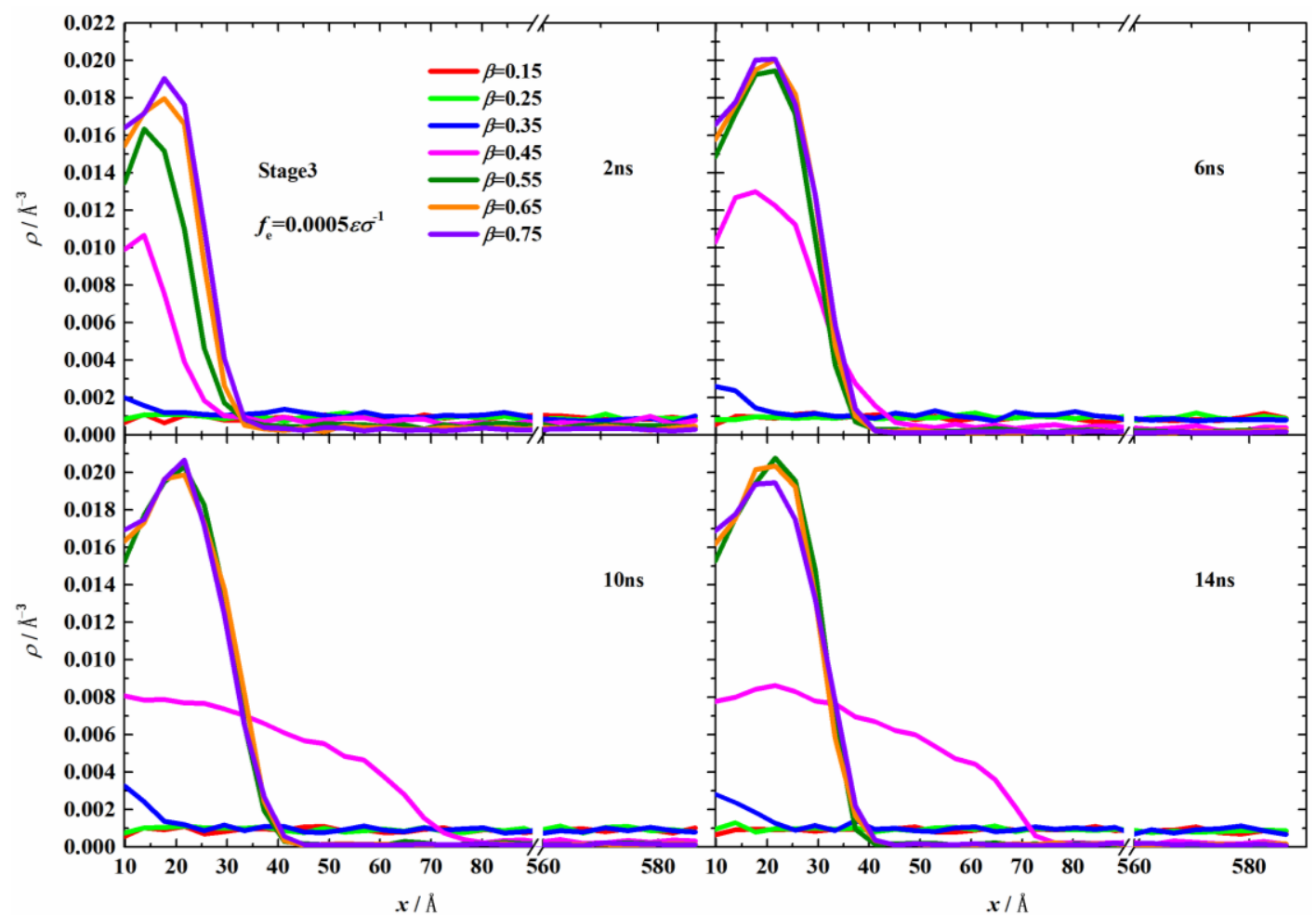

Figure 8. Transient density profiles with different $\beta$ and $f_{\mathrm{e}}=0.0005 \varepsilon \sigma^{-1}$ during stage 3 .

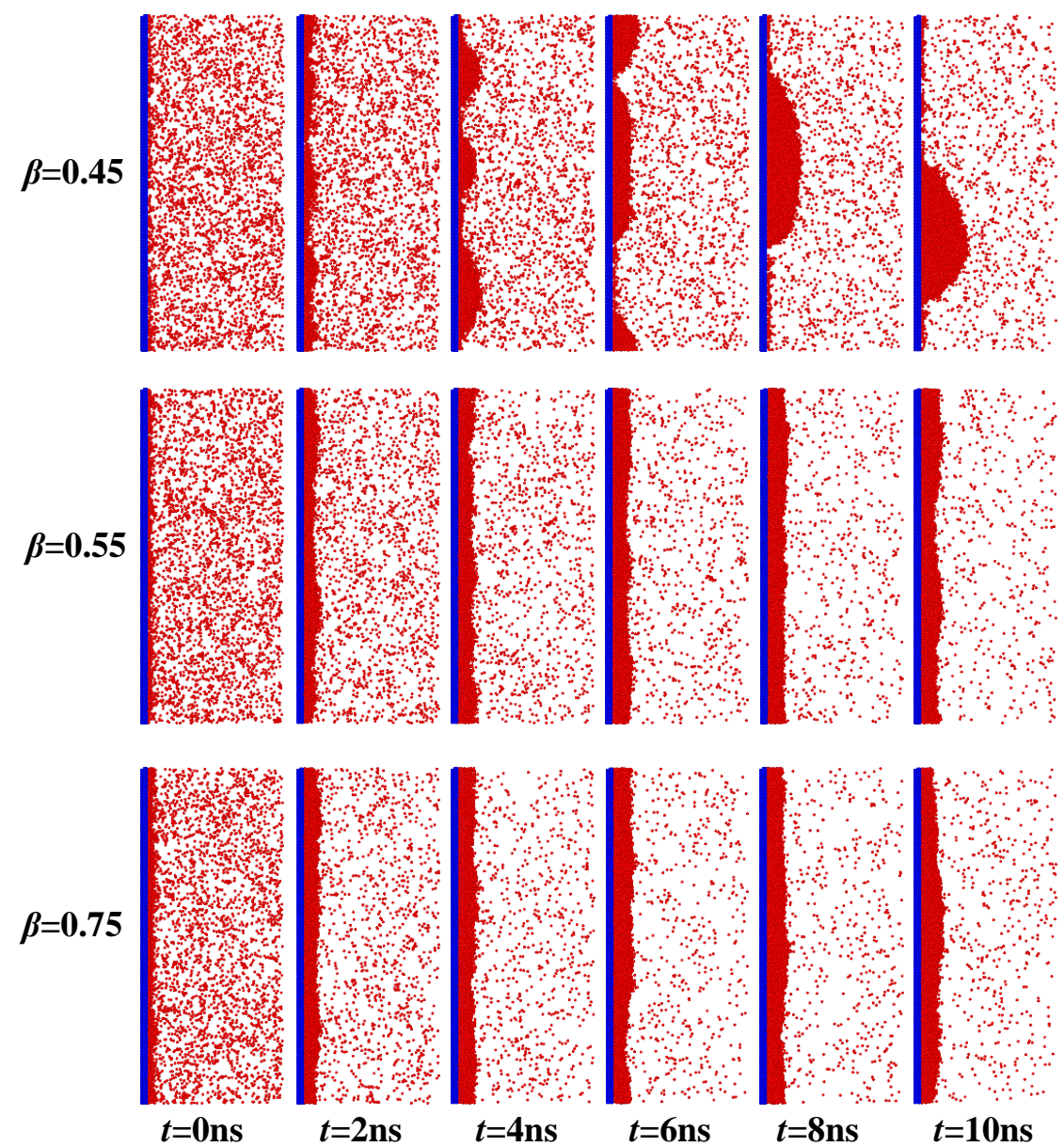

Figure 9. Transient snapshots with different $\beta$ and $f_{\mathrm{e}}=0.0005 \varepsilon \sigma-1$ during stage 3 . 


\subsection{Interfacial heat transfer}

\subsubsection{On surface with fixed wettability under different external force fields}

Surface condensation is influenced by solid-fluid interfacial heat transfer. In order to understand the effect of external force field on condensation, we measured the interfacial thermal resistance $(R)$ between the fluid and wall surface in stage 3 . The interfacial thermal resistance is determined as $R=\Delta T / Q A$, where $\Delta T$ is the temperature difference between the wall surface and the first layer of fluid molecules adjacent to it; $Q$ is the heat transfer rate, calculated as the slope of accumulated energy $(E)$ against time; $A$ is the heat transfer area. From Figs. 10 and 11 , it is found that if there is no condensation $\left(f_{\mathrm{e}} \geq 0.0005 \varepsilon \sigma^{-1}\right)$, the slope of $E$ remains unchanged, namely $Q$ keeps constant. Whereas, if condensation occurs $\left(f_{\mathrm{e}}<0.0005 \varepsilon \sigma^{-}\right.$ $\left.{ }^{1}\right), Q$ drops with time, which qualitatively means that the condensation intensity decreases with time. Variations of $R$ under different $f_{\mathrm{e}}$ are given in Fig. 12, which indicates that $R$ generally holds for all cases. It strongly verifies that $R$ is the inherent property for a given couple of solid and fluid (liquid or vapor), and independent of the transient heat transfer processes with different $f_{\mathrm{e}}$. In other words, once the solid wall and fluid (liquid or vapor) are primarily fixed, $R$ is simultaneously determined and fixed regardless of different transient heat transfer rates (see $f_{\mathrm{e}}=0 \sim 0.0004 \varepsilon \sigma^{-1}$ for solid-liquid cases with condensation and $f_{\mathrm{e}}=0.0006 \varepsilon \sigma^{-1} \sim 0.0007 \varepsilon \sigma^{-}$

${ }^{1}$ for solid-vapor cases without condensation occurring in Fig. 12). On the other hand, also note that the solid-liquid and solid-vapor interfacial thermal resistances are significantly different in nature, therefore, when there exists liquid-vapor phase-change, e.g. condensation in the present work, $R$ no longer holds but changes gradually and correspondingly, e.g. from the high value of solid-vapor interfacial thermal resistance to the low value of solid-liquid interfacial thermal resistance with notably transitional characteristics in the present work (see $f_{\mathrm{e}}=0 \sim 0.0002 \varepsilon \sigma^{-1}$ cases before $t=4 \mathrm{~ns}, f_{\mathrm{e}}=0.0003 \varepsilon \sigma^{-1}$ case before $t=8 \mathrm{~ns}, f_{\mathrm{e}}=0.0004 \varepsilon \sigma^{-1}$ case before $t=10 \mathrm{~ns}$ and $f_{\mathrm{e}}=0.0005 \varepsilon \sigma^{-1}$ case for whole simulation time-span in Fig. 12). 
In order to further investigate how $f_{\mathrm{e}}$ determines the surface condensation, two intentionallydesigned cases of $f_{\mathrm{e}}=0.0005 \varepsilon \sigma^{-1}$ near-wall $\left(f_{\mathrm{e}}\right.$ is just exerted on fluid molecules on near-wall region where the width of $x$ direction is $r_{\mathrm{c}}$, shown in Fig. 1) and $f_{\mathrm{e}}=0.0005 \varepsilon \sigma^{-1}$ main $\left(f_{\mathrm{e}}\right.$ is exerted on every fluid molecule except that situated on near-wall region, namely main-flow region) are carried out. Informative and distinct results are acquired that the variations of $E, Q$ and $R$ for $f_{\mathrm{e}}=0.0005 \varepsilon \sigma^{-1}$ near-wall case are completely in accord with $f_{\mathrm{e}}=0$ case whereas those for $f_{\mathrm{e}}=0.0005 \varepsilon \sigma^{-1}$ main case agree well with $f_{\mathrm{e}}=0.0005 \varepsilon \sigma^{-1}$ case (see Figs. 10-12). The results clearly show that the exertion of external force field on all fluid molecules is equivalent to that on the non-near-wall fluid molecules in the mainstream. This means that the external force field influences the surface condensation, including the onset and intensity, through the fluid bulk rather than the solid-fluid interface. Referring to the concepts in our previous work that the influencing factors to micro/nanofluidic phenomena can be categorized as either boundary or bulk factors [19], the external force field is clearly a bulk factor, rather than a boundary factor, to surface condensation. On the other hand, it is notable that the external force field does show indirect influence on the solid-fluid interfacial thermal resistance through direct influence on the surface condensation resulting from the generation of dissipated heat.

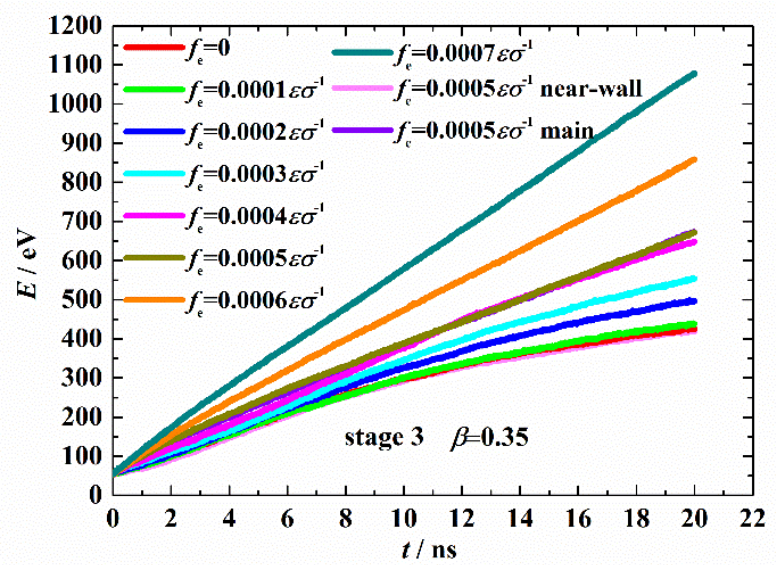

Figure 10. Time evolution of total heat transfer accumulation on surface with $\beta=0.35$ under different external force during stage 3 . 


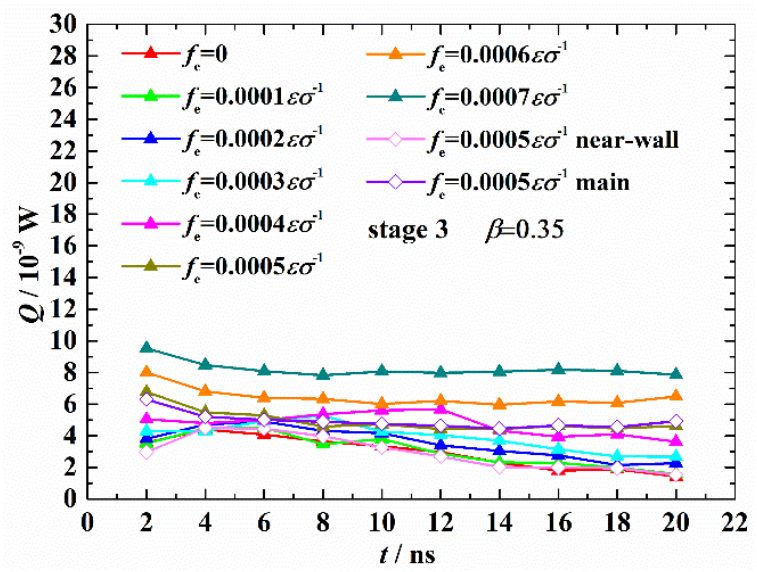

Figure 11. Time evolution of heat transfer rate on surface with $\beta=0.35$ under different external force during stage 3 .

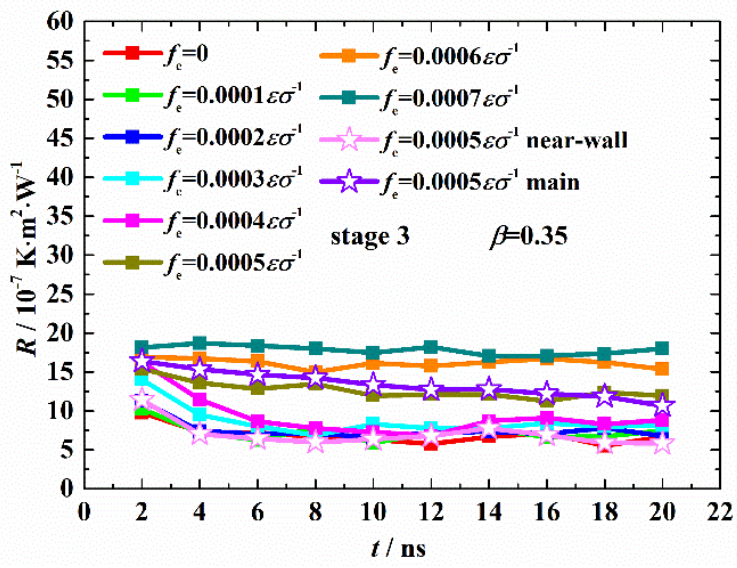

Figure 12. Time evolution of interfacial thermal resistance $(R)$ on a surface with $\beta=0.35$ under different external force fields during stage 3 .

\subsubsection{On surfaces with different wettabilities under a fixed external force field}

Similarly, the variations of $E, Q$ and $R$ against time with different $\beta$ and $f_{\mathrm{e}}=0.0005 \varepsilon \sigma^{-1}$ are statistically obtained during stage 3, as shown in Figs. 13-15. We find that $Q$ stays at low value $\left(<7 \times 10^{-9} \mathrm{~W}\right)$ and almost remains the same in non-condensation $(\mathrm{NC})$ cases $(\beta \leq 0.35)$. For condensation cases $(\beta>0.35), Q$, which indicates the condensation intensity, increases with increasing $\beta$ at the initial time but deceases with time, as shown in Figs.13-14. Due to the high condensation intensity, $Q$ drops more dramatically with increasing $\beta$. For the dropwise condensation (DWC) case $(\beta=0.45)$, the condensation process does not cease during our simulation time, whereas, for the filmwise condensation (FWC) cases $(\beta>0.45)$, the condensation process ceases before $t=14 \mathrm{~ns}$. As for $R$, it is highly sensitive to $\beta$. Generally, $R$ decreases with increasing $\beta$ following a nonlinear law [28]. When $\beta$ is relatively large, the 
difference of $R$ between cases with different $\beta$ is much smaller, as shown in Fig.16. All these results strongly suggest that the surface wettability exerts a significant effect on surface condensation.

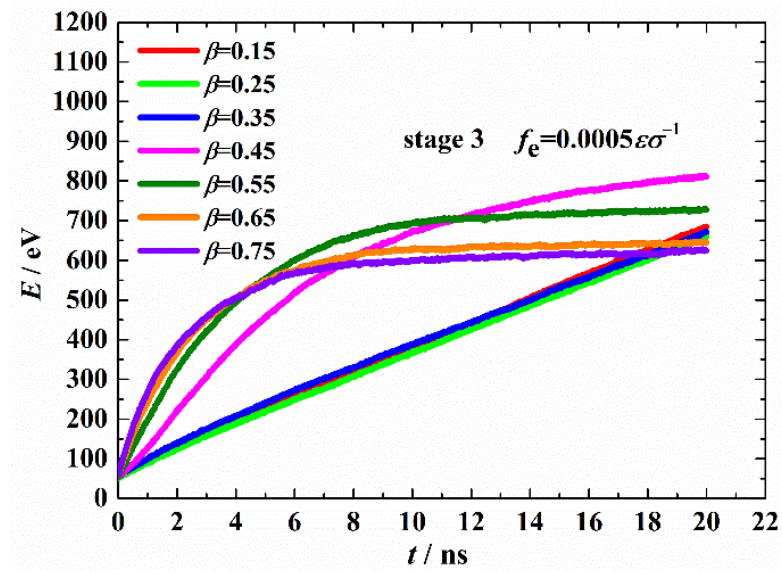

Figure 13. Time evolution of total heat transfer accumulation with different surface wettabilities under $f_{\mathrm{e}}=$ $0.0005 \varepsilon \sigma^{-1}$ during stage 3 .

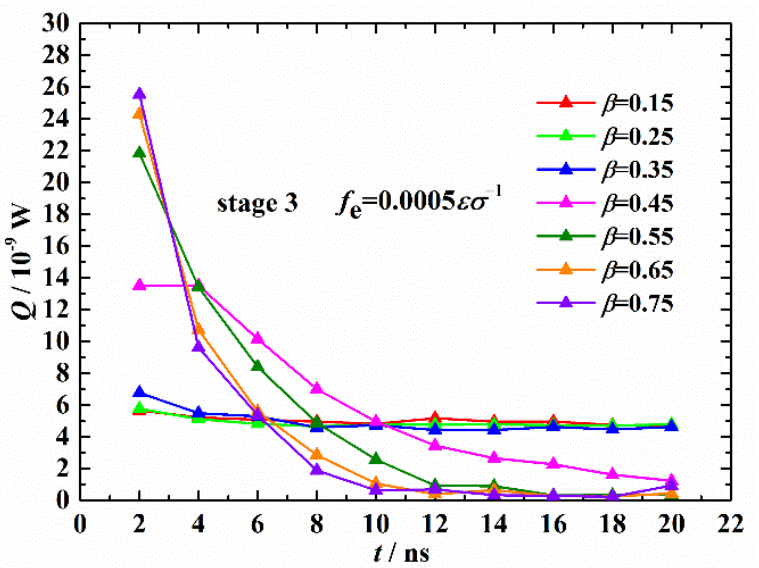

Figure 14. Time evolution of heat transfer rate with different surface wettabilities under $f_{\mathrm{e}}=0.0005 \varepsilon \sigma^{-1}$ during stage 3 .

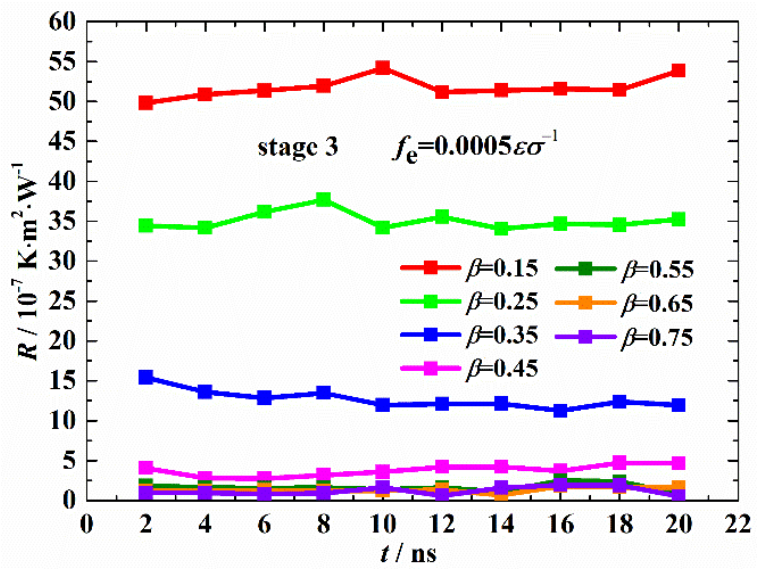

Figure 15. Time evolution of interfacial thermal resistance $(R)$ with different surface wettabilities under $f_{\mathrm{e}}=$ $0.0005 \varepsilon \sigma^{-1}$ during stage 3 . 


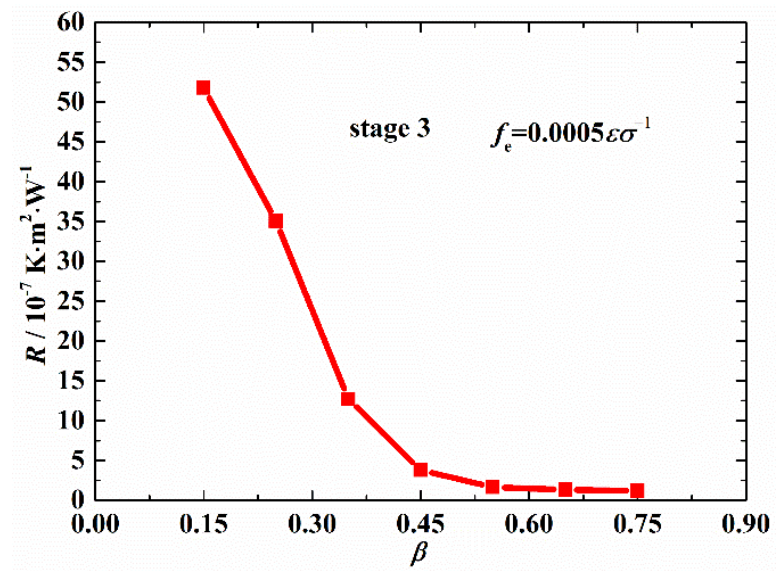

Figure 16. Interfacial thermal resistance $(R)$ against different surface wettabilities under $f_{\mathrm{e}}=0.0005 \varepsilon \sigma^{-1}$ during stage 3.

\subsection{Energy conversion}

To reveal the fundamental mechanism of effect of $f_{\mathrm{e}}$ on condensation from an energy point of view, energy conversion analysis has been carried out based on energy balance. The internal energy $(U)$, microscopically defined as the sum of thermal kinetic energy and potential energy, can be employed as the indicator of whether condensation/cooling occurs and how intensive it is. Figures 17 and 18 show the time-evolutions of changes in $U$ for different cases during stage 3. It is seen that for NC cases, $U$ slightly decreases at the initial time, then gradually converges to a certain value (marked in Figs. 17 and 18) after about $10 \mathrm{~ns}$. The convergence value increases with increasing $f_{\mathrm{e}}$ and decreasing $\beta$. For condensation cases, $U$ shows dramatic change when $f_{\mathrm{e}}=0 \sim 0.0004 \varepsilon \sigma^{-1}$ and $\beta=0.45 \sim 0.75$. It is also seen that $U$ changes more dramatically for FWC compared with DWC cases, which indicates that FWC is more intensive than DWC. All FWC cases almost cease after about $10 \mathrm{~ns}$ and $U$ converges to a certain value with slight difference between different cases. On the contrary, DWC cases apparently last after $10 \mathrm{~ns}$. Due to the dissipated heating effect of $f_{\mathrm{e}}$, vapor molecules are superheated, and the degree of superheating, as well as the initial values of $U$ before cooling, increases with increasing $f_{\mathrm{e}}$ and decreasing $\beta$. Generally, when the surface is cooled, $U$ decreases correspondingly. Note that this is a pure cooling process without condensation. Then, if $U$ stops decreasing and holds afterward, no condensation occurs. If $U$ continues to decrease dramatically, condensation 
occurs. Moreover, the more dramatically $U$ changes, the more intensive the condensation and more likely the filmwise condensation. It is clearly shown from Figs. 17 and 18 that both $f_{\mathrm{e}}$ and $\beta$ can significantly influence the nano-confined surface condensation.

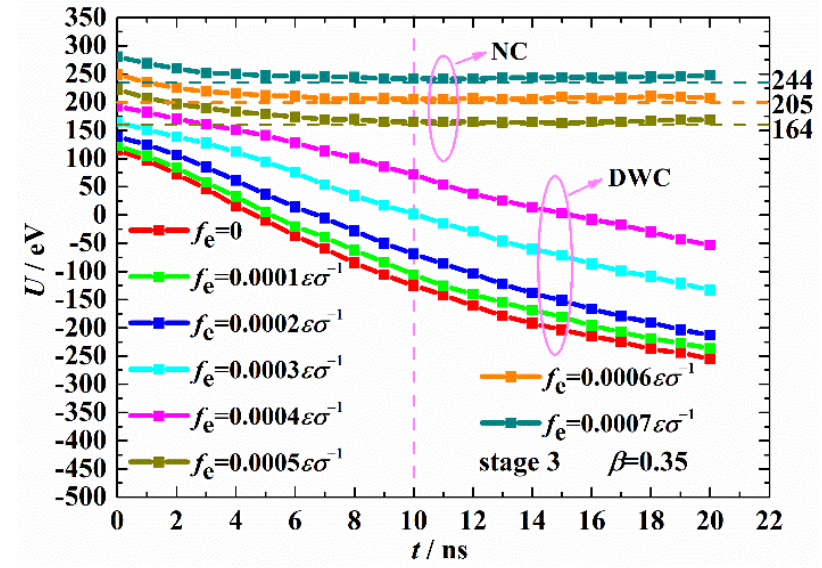

Figure 17. Time evolution of the internal energy $(U)$ with $\beta=0.35$ under different external forces during stage 3

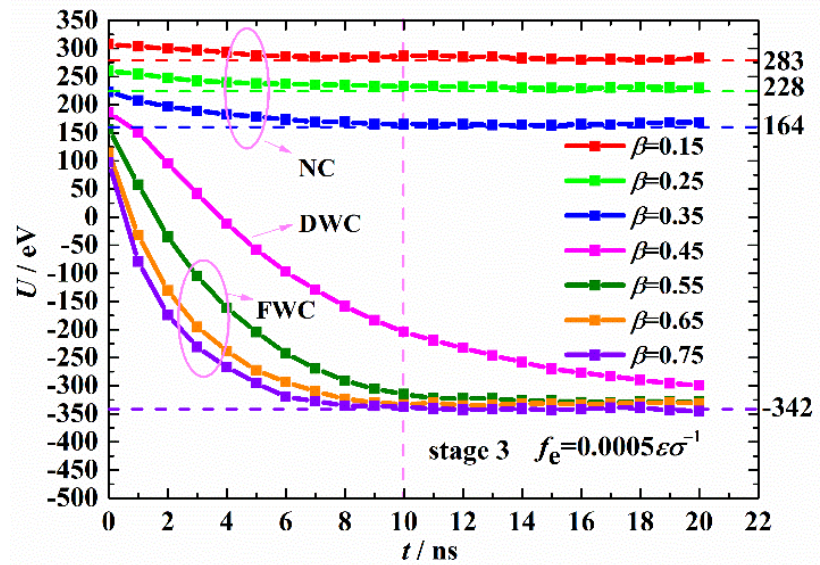

Figure 18. Time evolution of the internal energy $(U)$ with different surface wettabilities under $f_{\mathrm{e}}=0.0005 \varepsilon \sigma^{-1}$ during stage 3 .

For NC cases, $U$ remains steady after $10 \mathrm{~ns}$, suggesting condensation is unlikely to occur.

Therefore, we carry out energy conversion analysis based on the following equation:

$$
E_{\mathrm{Q}}=\Delta U+\Delta E_{\mathrm{K}}+W
$$

in the time span of $t=0 \sim 10 \mathrm{~ns}$, where $\Delta U$ and $\Delta E_{\mathrm{K}}$ are the changes in internal energy and macroscopic kinetic energy; $W$ and $E_{\mathrm{Q}}$ are the work done by external force and heat transferred from the fluid to the wall. The quantitative results are tabulated in Tabs. 2 and 3. To verify the energy balance, $E_{\mathrm{Q}}$ and $\Delta U+\Delta E_{\mathrm{K}}+W$ are compared with the relative deviations (RDs). It is found that almost all RDs are within $\pm 4 \%$, which guarantees the energy balance in Eq. 2 
considering the statistical fluctuation. Generally, no matter for the cases with same $\beta$ and different $f_{\mathrm{e}}$ or with different $\beta$ and same $f_{\mathrm{e}}$, the energy conversions obey the unified mechanism. Fig. 19 schematically illustrates the energy conversions based on Eq. 2.

For condensation cases ( $\operatorname{see} f_{\mathrm{e}}=0 \sim 0.0003 \varepsilon \sigma^{-1}$ in Tab. 2 and $\beta=0.45 \sim 0.75$ in Tab. 3), it is found that the heat transferred from fluid to solid mainly comes from $\Delta U(60 \sim 100 \%)$, releasing latent heat through the phase-change process. On the other hand, two other sources contributing to $E_{\mathrm{Q}}$ are identified to be secondarily $W(0 \sim 36 \%)$ and thirdly $\Delta E_{\mathrm{K}}(0 \sim 11 \%)$. Note that the case of $f_{\mathrm{e}}=0.0004 \varepsilon \sigma^{-1}$ and $\beta=0.35$ does not fully follow the above regulation $\left(\Delta U, W\right.$ and $\Delta E_{\mathrm{K}}$ respectively contribute $37 \%, 61 \%$ and $2 \%$ ) due to its transitional condensation state, therefore not considered. When condensation occurs, the work done within the bulk fluid $(W)$ completely converts into heat and dissipated through the solid wall. Meanwhile, $E_{\mathrm{K}}$ decreases due to the frictional effect at the solid-liquid interface. The corresponding change in $E_{\mathrm{K}}\left(\Delta E_{\mathrm{K}}\right)$ finally converts into heat and dissipated through the solid wall. In principle, $\Delta E_{\mathrm{K}}$ and $W$, which both result from $f_{\mathrm{e}}$, are dissipated by the friction and viscosity, and finally lead to heating effect to the fluid as an internal heat source, which can be clearly supported by the fact that when $f_{\mathrm{e}}$ is removed $\left(f_{\mathrm{e}}=0\right)$, both $\Delta E_{\mathrm{K}}$ and $W$ disappear simultaneously, as seen in Tab. 2 .

However, for non-condensation cases, either very strong viscous and frictional dissipations heating effect due to large $f_{\mathrm{e}}$ (see $f_{\mathrm{e}}=0.0005 \varepsilon \sigma^{-1} \sim 0.0007 \varepsilon \sigma^{-1}$ in Tab. 2) or very large interfacial thermal resistance due to small $\beta$ (see $\beta=0.15 \sim 0.35$ in Tab. 3) keeps the bulk vapor temperature higher than the saturation temperature and denies the occurrence of condensation though $U$ decreases a little, releasing sensible heat, due to the cooling of vapor molecules by the solid wall surface. Meanwhile, $W$ due to $f_{\mathrm{e}}$ mostly converts into dissipated heat while slightly contributes to the increase in $E_{\mathrm{K}}$. Therefore, it is found that $W$ and $\Delta U$ respectively contribute firstly (86 94\%) and secondly (7 17\%) to $E_{\mathrm{Q}}$ whereas $\Delta E_{\mathrm{K}}$ gives negative contribution $(-1 \sim-3 \%)$. 
Table 2. Energy conversions for cases with $\beta=0.35$ under different external force fields $(t=0 \sim 10 \mathrm{~ns})$.

\begin{tabular}{ccccccc}
\hline \hline$f_{\mathrm{e}}$ & $\Delta U$ & $\Delta E_{\mathrm{K}}$ & $W$ & $\Delta U+\Delta E_{\mathrm{K}}+W$ & $E_{\mathrm{Q}}$ & $\mathrm{RD}$ \\
$\mathrm{eV} \cdot \AA^{-1}$ & $\mathrm{eV}$ & $\mathrm{eV}$ & $\mathrm{eV}$ & $\mathrm{eV}$ & $\mathrm{eV}$ & $\%$ \\
\hline 0 & -224.2 & 0.0 & 0.0 & -224.2 & -227.5 & -1.43 \\
$0.0001 \varepsilon \sigma^{-1}$ & -222.5 & -2.7 & -11.9 & -237.1 & -237.5 & -0.19 \\
$0.0002 \varepsilon \sigma^{-1}$ & -189.8 & -9.0 & -46.8 & -245.6 & -249.8 & -1.70 \\
$0.0003 \varepsilon \sigma^{-1}$ & -173.6 & -13.6 & -104.1 & -291.4 & -301.1 & -3.30 \\
$0.0004 \varepsilon \sigma^{-1}$ & -111.3 & -5.2 & -181.4 & -297.8 & -314.5 & -5.45 \\
$0.0005 \varepsilon \sigma^{-1}$ & -52.9 & 9.2 & -277.5 & -321.3 & -330.8 & -2.94 \\
$0.0006 \varepsilon \sigma^{-1}$ & -40.3 & 9.8 & -380.9 & -411.4 & -418.8 & -1.80 \\
$0.0007 \varepsilon \sigma^{-1}$ & -41.7 & 10.0 & -487.9 & -519.6 & -523.8 & -0.80 \\
\hline \hline
\end{tabular}

Table 3. Energy conversions for cases with different surface wettability under $f_{\mathrm{e}}=0.0005 \varepsilon \sigma^{-1}(t=0 \sim 10 \mathrm{~ns})$.

\begin{tabular}{ccccccc}
\hline \hline$\beta$ & $\Delta U$ & $\Delta E_{\mathrm{K}}$ & $W$ & $\Delta U+\Delta E_{\mathrm{K}}+W$ & $E_{\mathrm{Q}}$ & $\mathrm{RD}$ \\
- & $\mathrm{eV}$ & $\mathrm{eV}$ & $\mathrm{eV}$ & $\mathrm{eV}$ & $\mathrm{eV}$ & $\%$ \\
\hline 0.15 & -23.7 & 3.8 & -298.9 & -318.8 & -321.0 & -0.68 \\
0.25 & -28.2 & 6.1 & -288.2 & -310.3 & -313.0 & -0.86 \\
0.35 & -52.9 & 9.2 & -277.5 & -321.3 & -330.8 & -2.94 \\
0.45 & -383.5 & -66.7 & -164.6 & -614.8 & -613.1 & 0.27 \\
0.55 & -467.4 & -70.5 & -103.8 & -641.7 & -629.5 & 1.91 \\
0.65 & -449.6 & -57.9 & -76.7 & -584.2 & -570.2 & 2.42 \\
0.75 & -438.3 & -51.5 & -66.0 & -555.8 & -542.8 & 2.37 \\
\hline \hline
\end{tabular}


(a)

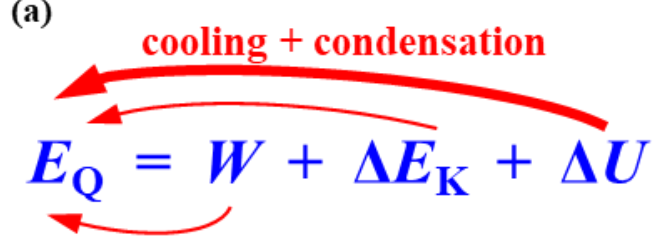

(b)

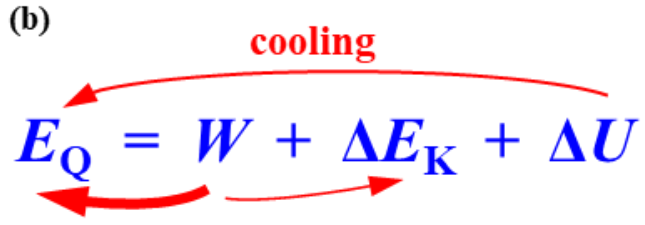

Figure 19. Energy conversion analyses for (a) condensation and (b) no-condensation cases (bold arrows indicate primary contributions).

Based on the above energy conservation analyses, the heat transferred from fluid to solid can be divided into two categories. One is the latent and sensible heats originated from the cooling and condensation, or pure cooling, of the fluid, which is represented by $Q_{\mathrm{c}}$. The other is the dissipated heat generated due to the existence of the external force field, which is represented by $Q_{\mathrm{f}}$. The proportional contributions of $Q_{\mathrm{c}}$ and $Q_{\mathrm{f}}$ in different cases based on $f_{\mathrm{e}}$ and $\beta$ are illustrated in Figs. 20 and 21, respectively. It is clearly seen that $Q_{\mathrm{c}}$ decreases and $Q_{\mathrm{f}}$ increases with increasing $f_{\mathrm{e}}$ and decreasing $\beta$. When $f_{\mathrm{e}}$ is small $(\beta=0.35)$ or $\beta$ is large $\left(f_{\mathrm{e}}=0.0005 \varepsilon \sigma^{-1}\right)$, condensation occurs in either FWC or DWC mode and the heat transferred from fluid to solid mainly comes from $Q_{\mathrm{c}}$. When $f_{\mathrm{e}}$ increases above a critical value of ca. $f_{\mathrm{cr}}=0.0004 \varepsilon \sigma^{-1}(\beta=0.35)$ or $\beta$ decreases below a critical value of ca. $\beta_{\mathrm{cr}}=0.45\left(f_{\mathrm{cr}}=0.0005 \varepsilon \sigma^{-1}\right)$, condensation never occurs and the heat transferred from fluid to solid wall surface mainly comes from $Q_{\text {f. }}$ It has been reported that the surface wettability is significant in determining the condensation mode and intensity. More importantly, it has not been reported that the existence of the external force field can postpone and even suppress the occurrence of condensation. Note that the surface wettability is normally regarded as a pretreating factor that cannot be easily and artificially changed during operation whereas the external force field is often a process factor that can be flexibly controlled in-situ, e.g. electrostatic and magnetic force fields. 


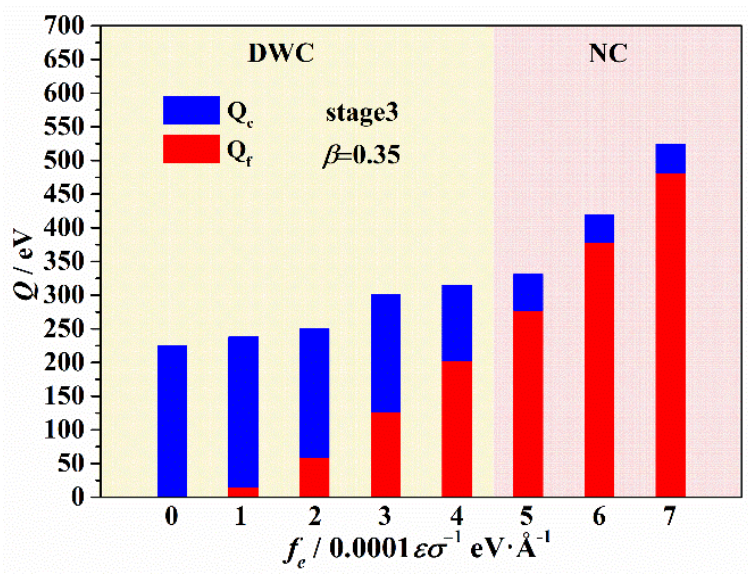

Figure 20. Different heat generation from 0 to $10 \mathrm{~ns}$ on surface with $\beta=0.35$ under different external force. The dropwise condensation (DWC) and no-condensation (NC) cases are shown in light yellow and red backgrounds.

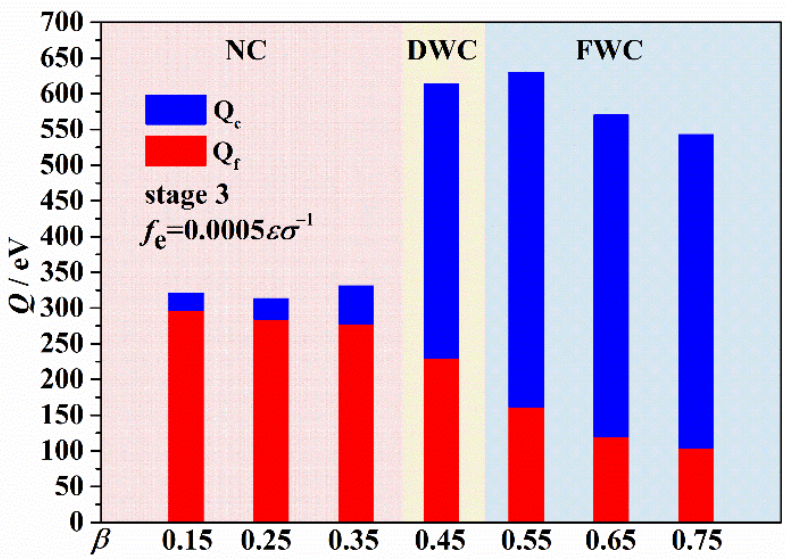

Figure 21. Different heat generation from 0 to $10 \mathrm{~ns}$ with different surface wettability under $f_{\mathrm{e}}=0.0005 \varepsilon \sigma^{-1}$. The no-condensation (NC), dropwise condensation (DWC) and filmwise condensation (FWC) cases are shown in light red, yellow and blue backgrounds.

\section{Conclusions}

In the present work, we investigate the dynamic behaviors of nano-confined surface condensation with different $\beta$ and $f_{\mathrm{e}}$ using MD simulations in the views of heat transfer and energy conversion. The simulation results show that both the velocity slip and temperature jump become larger with increasing $f_{\mathrm{e}}$ and decreasing $\beta$. Meanwhile, the existence of the velocity slip shows significant influence on the increase of fluid temperature. The density profiles for different $f_{\mathrm{e}}$ vary slightly on stage 2 . Contrastively, those for different $\beta$ show obvious difference and the density near the wall surface increases with increasing $\beta$. Asymmetric droplet is observed due to $f_{\mathrm{e}}$, which promotes the coalescence of droplets after 
nuclei form. Meanwhile, increasing $f_{\mathrm{e}}$ postpones or even suppresses the onset of surface condensation when it reaches a certain critical value $\left(f_{\text {cr }}=0.0005 \varepsilon \sigma^{-1}\right.$ for surface with $\left.\beta=0.35\right)$. Based on the heat transfer analysis, it can be found that $f_{\mathrm{e}}$, as a bulk factor, shows indirect effect on $R$ through direct influence on the surface condensation resulting from the generation of dissipated heat. In addition, when $R$ increases with decreasing $\beta$, the solid-vapor interfacial thermal resistance becomes significantly larger than the solid-liquid one.

Based on the energy balance analysis, for condensation cases, the heat transferred from fluid to solid mainly comes from the change in $U$, while for non-condensation cases, the dissipated heats are the major contributions to this heat. In all cases, $W$ mainly converts into dissipated heats, only except for the no-condensation case where a small proportion of $W$ converts into $E_{\mathrm{k}}$. We classify the heat transferred from fluid to solid into two categories, namely $Q_{\mathrm{c}}$ (due to condensation/cooling) and $Q_{\mathrm{f}}$ (due to $f_{\mathrm{e}}$ ). It is found that $Q_{\mathrm{f}}$ increases and dominates the total heat with increasing $f_{\mathrm{e}}$ and decreasing $\beta$. It is also found that $Q_{\mathrm{f}}$ plays an important role as the internal heat source and suppresses the further decrease of $U$, consequently the condensation is weakened or even inhibited.

\section{Acknowledgements}

The present work is supported by National Natural Science Foundation of China (51776196), the National Basic Research Program of China (973 Program) (2015CB251505), the Engineering and Physical Sciences Research Council (EPSRC) of the UK (EP/N020472/1, EP/N001236/1) and the Joint Ph.D. Studentship of China Scholarship Council (CSC) and Queen Mary University of London are acknowledged.

\section{Declarations of interest}

None 


\section{References}

[1]J. T. W. Yeow and J. P. M. She, Carbon nanotube-enhanced capillary condensation for a capacitive humidity sensor, Nanotechnology 17, 5441 (2006).

[2]D. Wilms, A. Winkler, P. Virnau, and K. Binder, Rounding of phase transitions in cylindrical pores, Phys. Rev. Lett. 10, 045701 (2010).

[3]S. S. Yoon and D. Y. Khang, Room-temperature chemical welding and sintering of metallic nanostructures by capillary condensation, Nano Lett. 16, 3550 (2016).

[4]L. Li, P. Ji, and Y. Zhang, Molecular dynamics simulation of condensation on nanostructured surface in a confined space, Appl. Phys. A 122, 496 (2016).

[5]E. Barsotti, S. P. Tan, S. Saraji, M. Piri, and J. H. Chen, A review on capillary condensation in nanoporous media: Implications for hydrocarbon recovery from tight reservoirs, Fuel 184, 344 (2016).

[6]J. Zhong, S. H. Zandavi, H. Li, B. Bao, A. H. Persad, F. Mostowfi, and D. Sinton, Condensation in OneDimensional Dead-End Nanochannels, ACS Nano 11, 304 (2016).

[7]J. C. T. Eijkel and A. Van Den Berg, Nanofluidics: what is it and what can we expect from it? Microfluidics and Nanofluidics, Microfluid. Nanofluid. 1, 249 (2005).

[8]R. B. Schoch, J. Han, and P. Renaud, Transport phenomena in nanofluidics, Rev. Mod. Phys. 80, 839 (2008). [9]K. Yasuoka, G. T. Gao, and X. C. Zeng, Molecular dynamics simulation of supersaturated vapor nucleation in slit pore, J. Chem. Phys. 112, 4279 (2000).

[10]K. T. Kholmurodov, K. Yasuoka, and X. C. Zeng, Molecular dynamics simulation of supersaturated vapor nucleation in slit pore. II. Thermostatted atomic-wall model, J. Chem. Phys. 114, 9578 (2001).

[11]W. Xu, Z. Lan, B. L. Peng, R. F. Wen, and X. H. Ma, Effect of surface free energies on the heterogeneous nucleation of water droplet: A molecular dynamics simulation approach, J. Chem. Phys. 142, 054701 (2015). [12]D. Suh, K. Yasuoka, and X. C. Zeng, Molecular dynamics simulation of heterogeneous nucleation on nanotube, RSC Adv. 5, 40953 (2015).

[13]D. Niu and G. H. Tang, The effect of surface wettability on water vapor condensation in nanoscale.

Scientific Reports, Sci. Rep. 6, 19192 (2016).

[14]Q. Sheng, J. Sun, Q. Wang, W. Wang, and H. S. Wang, On the onset of surface condensation: formation and transition mechanisms of condensation mode, Sci. Rep. 6, 30764 (2016).

[15]J. Sun and H. S. Wang, On the early and developed stages of surface condensation: competition mechanism between interfacial and condensate bulk thermal resistances, Sci. Rep. 6, 35003 (2016). 
[16]D. Niu and G. H. Tang, Molecular dynamics simulation of droplet nucleation and growth on a rough surface: revealing the microscopic mechanism of the flooding mode, RSC Adv. 8, 24517 (2018).

[17]B. H. Kim, A. Beskok, and T. Cagin, Viscous heating in nanoscale shear driven liquid flows. Microfluidics and Nanofluidics, Microfluid. Nanofluid. 9, 31 (2010).

[18]Z. Li, Surface effects on friction-induced fluid heating in nanochannel flows, Phys. Rev. E 79, 026312 (2009).

[19]J. Sun, W. Wang, and H. S. Wang, Dependence of nanoconfined liquid behavior on boundary and bulk factors, Phys. Rev. E 87, 023020 (2013).

[20]J. Sun, W. Wang, and H. S. Wang, Dependence between velocity slip and temperature jump in shear flows, J. Chem. Phys. 138, 234703 (2013).

[21]J. Sun and H. S. Wang, Self-shedding and sweeping of condensate on composite nano-surface under external force field: enhancement mechanism for dropwise and filmwise condensation modes, Sci. Rep. 7, 8633 (2017).

[22]R. Angélil, J. Diemand, K. K. Tanaka, and H. Tanaka, Properties of liquid clusters in large-scale molecular dynamics nucleation simulations, J. Chem. Phys. 140, 074303 (2014).

[23]K. K. Tanaka, A. Kawano, and H. Tanaka, Molecular dynamics simulations of the nucleation of water: Determining the sticking probability and formation energy of a cluster. The Journal of Chemical Physics, J. Chem. Phys. 140, 114302 (2014).

[24]T. Kraska, Molecular-dynamics simulation of argon nucleation from supersaturated vapor in the NVE ensemble, J. Chem. Phys. 124, 054507 (2006).

[25]J. Wedekind, D. Reguera, and R. Strey, Finite-size effects in simulations of nucleation, J. Chem. Phys. 125, 214505 (2006).

[26]S. Plimpton, Fast parallel algorithms for short-range molecular dynamics, J. Comput. Phys. 117, 1 (1995).

[27] Supplemental Material for Figs S1-S8.

[28]L. Xue, P. Keblinski, S. R. Phillpot, S. R. Phillpot, S. U.-S. Choi, and J. A. Eastman, Two regimes of thermal resistance at a liquid-solid interface, J. Chem. Phys. 118, 337 (2003). 